Pacific Journal of Mathematics

A SPECTRAL THEORY FOR A CLASS OF LINEAR
OPERATORS 


\title{
A SPECTRAL THEORY FOR A CLASS OF LINEAR OPERATORS
}

\author{
G. K. LEAF
}

In this paper we introduce a type of spectral theory for bounded operators in a Banach space. We shall focus most of our attention on the continuous spectrum, since the point spectrum, at least when it is isolated, can be handled by using the contour integral techniques developed by F. Riesz, E. R. Lorch, and N. Dunford and discussed in $[4,7]$.

In 1941, E. R. Lorch [6] treated a class of operators in a reflexive Banach space which are natural generalizations of unitary operators. By using ingenious methods, he was able to find invariant manifolds for these operators and constructed a spectral theory which in many respects is similar to that which is available for unitary operators. More recently, N. Dunford [2,3] has developed an extensive spectral theory for certan classes of operators in Banach spaces, and related work in this area has been done by F. Wolf [9] and others. However, Dunford's class of spectral operators does not contain the class studied by Lorch.

In this paper we will employ some of Dunford's techniques to obtain results which parallel those of Lorch [6]. In doing so, we are able to handle a larger class of operators than in [6], at least in the case where the spectrum is entirely continuous. Finally, we wish to point out that the results in Section 2 are not best possible.

1. Preliminary remarks. If $T$ is a bounded linear operator in a complex Banach space $X$, then $R(z ; T)$ will denote the resolvent operator $(z-T)^{-1}$ defined for $z$ in the resolvant set of $T$. When $T$ is understood, the notation $R(z)$ will be used in place of $R(z ; T)$. For any two points $z_{1}$ and $z_{2}$ in the resolvant set, $R$ satisfies the following relations:

(i) $R\left(z_{1}\right)-R\left(z_{2}\right)=\left(z_{2}-z_{1}\right) R\left(z_{1}\right) R\left(z_{2}\right)$, and

(ii) $R\left(z_{1}\right) R\left(z_{2}\right)=R\left(z_{2}\right) R\left(z_{1}\right)$.

One consequence of the above relations is the analyticity of the vectorvalued function $R(z) x$ on the resolvant set $\rho(T)$ for each vector $x$ in the space $X$. Since $R(z) x$ is a vector-valued analytic function on $\rho(T)$, it is natural to speak of analytic extensions of $R(z) x$. The

Received March 20, 1962. This paper is a part of the author's doctoral thesis at the University of Illinois under the direction and encouragement of R. G. Bartle. The author also wishes to acknowledge the financial aid given to him by the National Science Foundation. 
following definitions are due to Dunford $[3 ; \mathrm{p} .326]$.

1.1 Definition. An analytic extension of $R(z) x$ is a vector-valued function $f$ defined and analytic on an open set $\Delta(f)$ containing $\rho(T)$, and satisfying the equation $(z-T) f(z)=x$ for every $z$ in $A(f)$.

It is clear that if $f(z)$ is an analytic extension of $R(z) x$, then $R(z) x=f(z)$ for every $z$ in $\rho(T) . \quad R(z) x$ is said to have the singlevalued extension property provided that for every pair $f$ and $g$ of analytic extensions of $R(z) x$, we have $f(z)=g(z)$ for $z$ in the intersection of $\Delta(f)$ and $\Delta(g)$. The union of the sets $\Delta(f)$ as $f$ varies over all analytic extensions of $R(z) x$ is called the resolvent set for $x$ and is denoted by $\rho(x)$. The complement of $\rho(x)$ is called the spectrum of $x$, and is denoted by $\sigma(x)$. If $R(z) x$ has the single-valued extension property, then $R(z) x$ has a maximal single-valued analytic extension $x(z)$ with domain $\rho(x)$ and with $x(z)=R(z) x$ for $z$ in $\rho(T)$.

2. A class of operators with continuous spectrum. In this section $V$ will denote an invertible bounded linear operator satisfying the following two conditions:

(a) $\left\|V^{n}\right\|=o(|n|)$ as $n$ tends to $\pm \infty$.

(b) The spectrum of $V$ is purely continuous; that is, the point and residual spectrum are empty.

2.1 THEOREM. The spectrum of $V$ lies on the circumference of the unit circle.

Proof. The conclusion of this theorem depends on assumption (a). From (a) we have $\left\|V^{n}\right\| \leqq n$ and $\left\|V^{-n}\right\| \leqq n$ for all $n$ sufficiently large. Taking the $n$th root of both sides of each inequality, and passing to the limit, we see that the spectral radii of $V$ and $V^{-1}$ are both less than or equal to one.

It is convenient to obtain series expansions for $R(z)$ in each component of $\rho(V)$. If $|z|>1$, then from (a), it is easily verified that $\sum_{n=0}^{\infty} z^{-n-1} V^{n}$ converges in the uniform operator topology to a bounded, everywhere-defined operator satisfying the equation:

$$
(z-V)\left(\sum_{n=0}^{\infty} z^{-n-1} V^{n}\right)=\left(\sum_{0}^{\infty} z^{-n-1} V^{n}\right)(z-V)=I .
$$

Hence if $|z|>1$, then $R(z)=\sum_{0}^{\infty} z^{-n-1} V^{n}$.

For $|z|<1$, (a) will imply that $-\sum_{0}^{\infty} z^{n} V^{-n-1}$ converges in the uniform operator topology to a bounded, everywhere-defined operator satisfying the above resolvent equation. Hence for $|z|<1$, we have $R(z)=-\sum_{0}^{\infty} z^{n} V^{-n-1}$. 
The behavior of $R(z)$ as $z$ approaches $\sigma(V)$ will be needed. For any point $\xi=e^{i \lambda}$, the transversal segment through $\xi$ generated by $z=(1+s) e^{i \lambda},-s_{0} \leqq s \leqq s_{0}, 0<s_{0} \leqq 1 / 2$, will be denoted by $\Delta(\xi)$. The next result is proved by direct examination of the above expressions for the resolvent operator.

2.2 Theorem. For any $\xi=e^{i \lambda}$ we have

$$
|s|^{2}|| R\left((1+s) e^{i \lambda}, V\right) \| \leqq M_{1}
$$

for $0<|s| \leqq s_{0}$ where $0<s_{0} \leqq 1 / 2$ and $M_{1}$ is a constant.

Dunford [2; p. 564] has shown that if the spectrum of a bounded linear operator $T$ is nowhere dense in the complex plane, then for each vector $x$ in the space $X, R(z ; T) x$ has the single-valued extension property. Since the operator $V$ under consideration has its spectrum on the circumference of the unit circle, and so has nowhere dense spectrum, for each $x$ in $X$ we may speak of the single-valued analytic function $x(\cdot)$ which is the maximal extension of $R(z ; V) x$. The following results (cf. [2; p. 564]) are immediate consequences of the definition of $x(\cdot)$.

2.3 Lemma. For any $x$ and $y$ in $X$, we have:

(a) $\sigma(x+y) \leqq \sigma(x) \cup \sigma(y)$,

(b) $(\alpha x+\beta y)(z)=\alpha x(z)+\beta y(z)$ for $z$ in $\rho(x) \cap \rho(y)$,

(c) $\sigma(x)=\varnothing$ if and only if $x=0$.

If $T$ is any bounded linear operator and $\mathscr{F}(T)$ is the algebra of scalar-valued functions analytic in some neighborhood of $\rho(T)$, then there exists an algebraic homomorphism of $\mathscr{F}(T)$ into the algebra $B(X)$ of all bounded linear operators on $X$. The homomorphism (cf. [7; ch. 5]) is given by

$$
f(T)=\frac{1}{2 \pi i} \int_{o} f(z) R(z ; T) d z, \quad f \text { in } \mathscr{F}(T),
$$

where $C$ is the boundary of an open set $D$ containing $\rho(T)$ and such that $D \cup C$ is contained in the domain of analyticity of $f$ and $C$ consists of a finite number of positively-oriented nonintersecting Jordan curves. In the case of the operator $V$, for each $f$ in $\mathscr{F}(V), C$ may be chosen to be the oriented boundary of some annular region containing the circumference of the unit circle. Let $f$ be in $\mathscr{F}(V)$ with domain of analyticity $\Delta(f)$; let $D=\left\{z: r_{1}<|z|<r_{2}, r_{1}<1<r_{2}\right\}$ be a region such that the boundary $C$ together with $D$ is contained in $\Delta(f)$. In addition, let $\xi$ be any point on the circumference of the unit circle with $\Delta(\xi)$ denoting the transversal segment through $\xi$ con- 
necting the inner and outer boundaries of $D$. In general the integral of $f(z) R(z) x$ along the transversal $\Delta(\xi)$ does not exist due to the possible unboundedness of $R(z) x$ on $\Delta(\xi)$; however, the restricted behavior of $R(z) x$ on $\Delta(\xi)$ implied by Theorem 2.2 will permit us to define the integral for a suitable choice of $f$. For $0 \leqq \lambda_{1}<\lambda_{2}<2 \pi$, let $C\left(\lambda_{1}, \lambda_{2}\right)$ denote the contour consisting of the $\operatorname{arcs} A B, B C, C D$, and $D A$ where $A, B, C$, and $D$ are given by: $A=(1-t) e^{i \lambda_{2}}, B=(1-t) e^{i \lambda_{1}}$, $C=(1+t) e^{i \lambda_{1}}$, and $D=(1+t) e^{i \lambda_{2}}$. The $\operatorname{arcs} A B$ and $C D$ are subarcs of circles centered at the origin of radii $1-t$ and $1+t$ respectively with $0<t \leqq 1 / 2$. The arcs $B C$ and $D A$ are the transversal segments through $\xi_{1}=e^{i \lambda_{1}}$ and $\xi_{2}=e^{i \lambda_{2}}$ respectively. The contour $C\left(\lambda_{1}, \lambda_{2}\right)$ is positively-oriented in the counter-clockwise direction. The complementary contour $C\left(\lambda_{2}, \lambda_{1}\right)$ consists of the subarcs $D C$ and $B A$ together with the transversals $C B$ and $A D$. The closed subarc of the circumference of the unit circle consisting of the points $\xi=e^{i \lambda}$ such that $\lambda_{1} \leqq \lambda \leqq$ $\lambda_{2}$ will be denoted by $\left[\lambda_{1}, \lambda_{2}\right]$ or $\left[\xi_{1}, \xi_{2}\right]$ whichever is more convenient. The closure of the complement of $\left[\lambda_{1}, \lambda_{2}\right]$ with respect to the circumference of the unit circle will be denoted by $\left[\lambda_{2}, \lambda_{1}\right]$ or $\left[\xi_{2}, \xi_{1}\right]$. The following two theorems, being specializations of theorems due to Dunford [2; 586], are stated without proof. It should be noted that integrals of the type to be discussed in the following theorems were first used by E. R. Lorch, Return to the Self-Adjoint Transformation, Szeged Acta, 1950.

2.4 THeOREM. Let $F(z)$ be analytic in the closed annulus $1-t \leqq$ $|z| \leqq 1+t, 0<t \leqq 1 / 2$. For $\xi_{1}=e^{i \lambda_{1}}$ and $\xi_{2}=e^{i \lambda_{2}}$ with $0 \leqq \lambda_{1}<$ $\lambda_{2}<2 \pi$, let $C\left(\lambda_{1}, \lambda_{2}\right)$ be the contour defined in the preceding discussion. Then

$$
J\left(\lambda_{1}, \lambda_{2} ; F\right)=\frac{1}{2 \pi i} \int_{\sigma\left(\lambda_{1}, \lambda_{2}\right)} F(z)\left(z-\xi_{1}\right)^{2}\left(z-\xi_{2}\right)^{2} R(z) d z
$$

exists as a Riemann integral, and is independent of $t$ provided $F(z)$ $i s$ analytic in the closed annulus determined by $t$. In addition, the spectrum $\sigma\left(J\left(\lambda_{1}, \lambda_{2} ; F\right) x\right)$ is contained in the intersection of $\left[\lambda_{1}, \lambda_{2}\right]$ and $\sigma(x)$. Furthermore, $\lim J\left(\lambda_{1}, \lambda_{2} ; F\right)=0$ as $\lambda_{2}-\lambda_{1}$ approaches 0 from above. If $F(z) \equiv 1$ then $J\left(\lambda_{1}, \lambda_{2} ; F\right)$ will be denoted by $J\left(\lambda_{1}, \lambda_{2}\right)$.

2.5 THEOREM. For every $\xi_{1}=e^{i \lambda_{1}}$ and $\xi_{2}=e^{i \lambda_{2}}$ the set $\{x: \sigma(x) \subseteq$ $\left.\left[\lambda_{1}, \lambda_{2}\right]\right\}$ is a closed linear manifold.

In the following theorems, the assumption that the spectrum is purely continuous will be used.

2.6 Lemma. For any $\xi=e^{i \lambda}$, the range of the operator $(V-\xi)^{2}$ 
is dense in the space $X$.

Proof. Let $A(M)$ denote the range of an operator $A$ acting on a linear manifold $M$, and $\operatorname{cl}(M)$ the closure of $M$. It is obvious that $(V-\xi)^{2}(X) \subseteq(V-\xi)(X)$. On the other hand, if $M=(V-\xi)(X)$, then since $\operatorname{cl}(M)=X$, we have:

$$
(V-\xi)(X)=(V-\xi)(\operatorname{cl}(M)) \subseteq \operatorname{cl}((V-\xi)(M))=\operatorname{cl}\left((V-\xi)^{2}(X)\right) .
$$

We now obtain a basic decomposition of the space $X$ relative to $V$.

2.7 THEOREM. Let $\xi_{1}, \xi_{2}, \cdots, \xi_{n}$ be points on the circumference of the unit circle, and let $W$ denote the range of the operator $\left(V-\xi_{1}\right)^{2}\left(V-\xi_{2}\right)^{2} \cdots\left(V-\xi_{n}\right)^{2}$; then

(i) $W$ is dense in the space $X$, and

(ii) for any vector $y$ in $W$ there is a unique decomposition $y=$ $y_{1}+y_{2}+\cdots+y_{n}$ with $\sigma\left(y_{k}\right) \subseteq\left[\xi_{k}, \xi_{k+1}\right]$, where we have set $\xi_{n+1}=\xi_{1}$.

Proof. Part (i) is obtained from Lemma 2.6 by induction. In order to prove part (ii), we note that by the operational calculus

$$
y=\left(V-\xi_{1}\right)^{2} \cdots\left(V-\xi_{n}\right)^{2} x=\frac{1}{2 \pi i} \int_{O}\left(z-\xi_{1}\right)^{2} \cdots\left(z-\xi_{n}\right)^{2} R(z) x d z,
$$

where $C$ is the oriented boundary of the region

$$
D=\{z: 1-t<|z|<1+t, 0<t \leqq 1 / 2\} .
$$

Using Theorem 2.2, we write the above integral as the sum of $n$ integrals in the following way:

$$
\begin{aligned}
y= & \frac{1}{2 \pi i} \int_{\sigma\left(\xi_{1}, \xi_{2}\right)}\left(z-\xi_{1}\right)^{2} \cdots\left(z-\xi_{n}\right)^{2} R(z) x d z+\cdots \\
& +\frac{1}{2 \pi i} \int_{\sigma\left(\xi_{n}, \xi_{1}\right)}\left(z-\xi_{1}\right)^{2} \cdots\left(z-\xi_{n}\right)^{2} R(z) x d z .
\end{aligned}
$$

Setting

$$
y_{k}=\frac{1}{2 \pi i} \int_{\sigma\left(\xi_{k}, \xi_{k+1}\right)}\left(z-\xi_{1}\right)^{2} \cdots\left(z-\xi_{n}\right)^{2} R(z) x d z \quad \text { for } k=1,2, \cdots, n,
$$

we have $y=y_{1}+y_{2}+\cdots+y_{n}$. From Theorem 2.4,

$$
\sigma\left(y_{k}\right) \subseteq\left[\xi_{k}, \xi_{k+1}\right] \cap \sigma(x) \text { for } k=1,2, \cdots, n .
$$

To show uniqueness, suppose

$$
y=y_{1}+y_{2}+\cdots+y_{n}=y_{1}^{\prime}+y_{2}^{\prime}+\cdots+y_{n}^{\prime},
$$


then

$$
y_{1}-y_{1}^{\prime}=\left(y_{2}^{\prime}-y_{2}\right)+\cdots+\left(y_{n}^{\prime}-y_{n}\right) \text {. }
$$

Hence by Lemma 2.3(a), we have

$$
\sigma\left(y_{1}-y_{1}^{\prime}\right) \subseteq\left[\xi_{1}, \xi_{2}\right] \cap\left[\xi_{2}, \xi_{1}\right]=\left\{\xi_{1}\right\} \cup\{\xi\} .
$$

Thus to show uniqueness, it suffices to show that $\sigma(u) \subseteq\{\xi\}$ implies that $u=0$. But it is easily seen that if $\sigma(u) \subseteq\{\xi\}$, then $(V-\xi)^{4} u=0$. However $(V-\xi)$ is by assumption one-to-one; hence $u=0$.

Before we proceed to the next theorem, we shall discuss a generalization of a result due to Lorch [6]. Although Lorch assumed that $\left\|V^{n}\right\| \leqq K$ for some constant $K$ and all integers $n$, we shall only assume that $\left\|V^{n}\right\|=o(|n|)$. This lemma is the key to the reconstruction of the operator $V$ from the spectral manifolds that will be introduced in Theorem 2.9.

2.8 Lemma. Let $y=(I-V)^{4} x$ and let $\varepsilon>0$ be given, then there exists a constant $A_{\varepsilon}$ such that

$$
\|(I-V) x\| \leqq(3 / 4) \varepsilon\|x\|+A_{\varepsilon}\|y\| .
$$

Proof. Since $\left\|V^{n}\right\|=o(|n|)$, there exists a constant $M>1$ such that $\left\|V^{n}\right\|<6 M n$ for $n=1,2, \cdots$. Thus if $\sigma_{n}=\sum_{0}^{n-1}(1-k / n) V^{k}$, we have $\left\|\sigma_{n}\right\| \leqq M\left(n^{2}+5\right)$. Now for any integers $n, m$, and $k$ and any vector $x$ in the space, we have:

$$
\begin{aligned}
\sigma_{k}\left((I-V)^{2} x\right) & =(I-V) x-k^{-1}\left(I-V^{k}\right) V x, \\
\sigma_{m}\left((I-V)^{3} x\right) & =(I-V)^{2} x-m^{-1}\left(I-V^{m}\right)(I-V) V x, \\
\sigma_{n}\left((I-V)^{4} x\right) & =(I-V)^{3} x-n^{-1}\left(I-V^{n}\right)(I-V)^{2} V x .
\end{aligned}
$$

For any integer $p$ and $i=0,1,2$, set $Q^{i}(p)=p^{-1}\left(I-V^{p}\right)(I-V)^{i} V$. Since $\left\|V^{n}\right\|=o(|n|)$, we have $\left\|Q^{i}(p)\right\|$ tending to zero as $p$ tends to infinity for $i=0,1,2$. Thus if $x$ is any vector in the space $X$, we have:

$$
\begin{array}{r}
\|(I-V) x\| \leqq Q^{0}(k)\|x\|+M\left(k^{2}+5\right) Q^{1}(m)\|x\|+M^{2}\left(k^{2}+5\right)\left(m^{2}+5\right) Q^{2}(n)\|x\| \\
+M^{3}\left(k^{2}+5\right)\left(m^{2}+5\right)\left(n^{2}+5\right)\left\|(I-V)^{4} x\right\| .
\end{array}
$$

Choosing $k, m$, and $n$, in succession, such that each of the first three terms is less than $\varepsilon / 4$ and setting $A_{\varepsilon}=M^{3}\left(k^{2}+5\right)\left(m^{2}+5\right)\left(n^{2}+5\right)$, we have the desired result. Using $\xi^{-1} V$, with $\xi$ on the circumference of the unit circle, in place of $V$ in this lemma would replace the identity operator $I$ apperaring in the desired inequality by $\xi I$.

2.9 Theorem. Given any $\varepsilon>0$ there exists $a \delta>0$ such that 
for any $\lambda$ with $0 \leqq \lambda \leqq 2 \pi$ and any vector $x$ in the closed linear manifold $L(\lambda)=\{x: \sigma(x) \leqq[\lambda, \lambda+\delta]\}$ we have

$$
\left\|\left(V-e^{i \lambda}\right) x\right\| \leqq \varepsilon\|x\| .
$$

Furthermore, let $n=[2 \pi / \delta]$ and let $\lambda_{k}=k \delta$ for $k=0,1, \cdots, n$; then the space $X$ is spanned by the manifolds $L\left(\lambda_{k}\right), k=0,1, \cdots, n$.

Proof. Let $\varepsilon>0$ be given, the choose $k, m$, and $n$ as was done in Lemma 2.8. Now choose a positive number $\varepsilon_{1}$ so small that $\varepsilon_{1} A_{\varepsilon}<$ $\varepsilon / 4$. For this choice of $\varepsilon_{1}$, choose a $\delta>0$ such that if $\xi=e^{i \lambda}$ is any point on the circumference of the unit circle, and if $\zeta=e^{i \mu}$ with $\mu=\lambda+\delta$, then

$$
\begin{gathered}
\left\|(V-\xi)^{2}(V-\zeta)^{2}-(V-\xi)^{4}\right\|<\varepsilon_{1} / 2, \\
\|J(\lambda, \mu)\|<\varepsilon_{1} / 2 .
\end{gathered}
$$

Inequality (2) is possible by Theorem 2.4, and the choice of $\delta$ is independent of $\lambda$. Set $L(\lambda)=\{x: \sigma(x) \leqq[\lambda, \mu]\}$; by Theorem $2.5, L(\lambda)$ is a closed linear manifold. Suppose $x$ is in $L(\lambda)$, then $J(\mu, \lambda) x=0$; hence,

$$
(V-\xi)^{2}(V-\zeta)^{2} x=J(\lambda, \mu) x+J(\mu, \lambda) x=J(\lambda, \mu) x .
$$

From (2) we then have:

$$
\left\|(V-\xi)^{2}(V-\zeta)^{2} x\right\|=\|J(\lambda, \mu) x\| \leqq \varepsilon_{1} / 2\|x\| .
$$

Thus

$$
\left\|(V-\xi)^{4} x\right\| \leqq \varepsilon_{1}\|x\| .
$$

By Lemma 2.8 and the choice of $\varepsilon_{1}$, we have

$$
\begin{gathered}
\|(V-\xi) x\| \leqq(3 / 4) \varepsilon\|x\|+A_{\varepsilon}\left\|(V-\xi)^{4} x\right\|<(3 / 4) \varepsilon\|x\| \\
+\varepsilon_{1} A_{\varepsilon}\|x\| \leqq(3 / 4) \varepsilon\|x\|+(\varepsilon / 4)\|x\|=\varepsilon\|x\| .
\end{gathered}
$$

Using the $\delta$ which was determined in the above discussion, we let $n$ be the greatest integer in $2 \pi / \delta$. Let $\lambda_{k}=k \delta$ and $\xi_{k}=e^{i \lambda_{k}}$ for $k=1,2, \cdots, n$ and set $\xi_{n+1}=2 \pi$; then from the first part of the theorem, we have $\left\|\left(V-\xi_{k}\right) x\right\| \leqq \varepsilon\|x\|$ for $x$ in $L\left(\lambda_{k}\right)$. For any $x$ in $X$ we may, by Theorem 2.7, approximate $x$ by a vector of the form $\left(V-\xi_{0}\right)^{2}\left(V-\xi_{1}\right)^{2} \cdots\left(V-\xi_{n}\right)^{2} y$. This vector may, in turn, be written as $y_{1}+y_{2}+\cdots+y_{n}$ with $y_{k}$ in $L\left(\lambda_{k}\right)$.

If $U$ is a unitary operator in a Hilbert space $H$, then the preceding theorem can be improved. Recall (cf. [5; p. 357]) that if $U$ is unitary, then there exists a resolution of the identity $E_{t}$ for $U$ such that for each continuous and periodic function $f$ on $[0,2 \pi]$ there 
corresponds an operator $U_{f}$ given by $U_{f}=\int_{0}^{2 \pi} f(t) d E_{t}$. Here the integral converges in the operator norm, and for each $x$ in $H,\left\|U_{f} x\right\|^{2}=$ $\int_{0}^{2 \pi}|f(t)|^{2} d\left\|E_{t} x\right\|^{2}$. If $\varepsilon>0$ and $\xi=e^{i \lambda}$ are given, let $\alpha=\lambda+\varepsilon$, then if $x$ is in the range of the projection $E_{\alpha}-E_{\lambda}$, we have $E_{t} x=0$ for $t \leqq \lambda$, and $E_{t} x=x$ for $\alpha \leqq t$. Thus

$$
\begin{aligned}
\left\|\left(U-e^{i \lambda}\right) x\right\|^{2} & =\int_{0}^{2 \pi}\left|e^{i t}-e^{i \lambda}\right|^{2} d\left\|E_{t} x\right\|^{2} \leqq \varepsilon^{2}\left(\left\|E_{\alpha} x\right\|^{2}-\left\|E_{\lambda-0} x\right\|^{2}\right) \\
& =\varepsilon^{2}\|x\|^{2} .
\end{aligned}
$$

If $\varepsilon>0$ is given, choose a partition $0=s_{0}<s_{1}<\cdots<s_{n+1}=2 \pi$ of $[0,2 \pi]$ with $s_{j+1}-s_{j}<\varepsilon$ for $j=1,2, \cdots, n+1$. Setting $L^{\prime}\left(s_{j}\right)=$ $\left(E_{s_{j+1}}-E_{s_{j}}\right)(X)$, and noting that $I=\sum_{j=1}^{n+1}\left(E_{s_{j+1}}-E_{s_{j}}\right)$ we see that $H=L^{\prime}\left(s_{1}\right) \oplus \cdots \oplus L^{\prime}\left(s_{n+1}\right)$ and from the above discussion, $\left\|\left(V-e^{i s_{j}}\right) y\right\| \leqq$ $\varepsilon\|y\|$ for each $y$ in $L^{\prime}\left(s_{j}\right)$. Thus in the case of a unitary operator, $H$ is the direct sum of the collection $L^{\prime}\left(s_{j}\right)$.

Numerous examples of bounded invertible operators, with any desired rate of growth for the iterates, may be obtained by considering the shift operator acting in certain sequence spaces. Such sequence spaces and analogous function spaces have been studied by A. Beurling, J. Wermer, and others. (cf. 8)

Let $\left\{p_{n}\right\},-\infty<n<\infty$, be a sequence of real numbers greater than or equal to one with $p_{0}=1$ and satisfing $p_{n+m} \leqq p_{n} p_{m}$ for all $m$ and $n$. Let $L$ denote the Banach space of all sequences $x=\left\{x_{n}\right\}$ such that $\sum_{n=-\infty}^{\infty} p_{n}\left|x_{n}\right|$ is finite and with this sum as the norm for the element $x$. Let $T$ be the shift operator defined on $L$ by $T x=y$ where $y=\left\{y_{k}\right\}$ and $y_{k}=x_{k-1}$. Then $T$ is a bounded invertible operator on $L$ and, for each $n,\left\|T^{n}\right\|=p_{n}$. In particular, setting $p_{n}=1+|n|^{\infty}$ for some fixed $\alpha$ such that $0<\alpha<1$, we obtain an operator $T$ for which $\left\|T^{n}\right\|=o(|n|)$ while $\left\|T^{n}\right\|$ is not bounded.

3. The resolving manifolds. In this section, a system $\left\{G_{\lambda}, F_{\lambda}\right\}$, $0 \leqq \lambda \leqq 2 \pi$, of pairs of closed linear manifolds is developed which has some of the properties of a resolution of the identity in the case of a unitary operator.

3.1 LEMma. Let $e^{i \lambda}$ and $e^{i \mu}$ be any two points on the circumference of the unit circle with $\lambda<\mu$ and denote the open subarc $\{z:|z|=1, z \notin$ $[\lambda, \mu]\}$ by $[\lambda, \mu]^{\prime}$; then $\left\{x+y: \sigma(x) \subseteq[\lambda, \mu], \sigma(y) \subseteq[\lambda, \mu]^{\prime}\right\}$ is dense in $X$.

Proof. Choose $\lambda_{1}<\lambda<\lambda_{2}<\mu_{1}<\mu<\mu_{2}$ : set

$$
F(z)=\left(z-e^{i \lambda_{1}}\right)^{2}\left(z-e^{i \lambda_{2}}\right)^{2}\left(z-e^{i \mu_{1}}\right)^{2}\left(z-e^{i \mu_{2}}\right)^{2},
$$


then using the notation of Theorem 2.4, we have for $x$ in $X$

(i) $\left(V-e^{i \lambda_{1}}\right)^{2}\left(V-e^{i \lambda_{2}}\right)^{2}\left(V-e^{i \mu_{1}}\right)^{2}\left(V-e^{i \mu_{2}}\right)^{2} x=J\left(\lambda_{1}, \lambda_{2}\right) x$ $+J\left(\lambda_{2}, \mu_{1}\right) x+J\left(\mu_{1}, \mu_{2}\right) x+J\left(\mu_{2}, \lambda_{1}\right) x$.

If $\lambda_{1}, \lambda_{2}$ tend to $\lambda$ and $\mu_{1}, \mu_{2}$ tend to $\mu$, then by Theorem 2.4, $J\left(\lambda_{1}, \lambda_{2}\right) x$ and $J\left(\mu_{1}, \mu_{2}\right) x$ both tend to zero, and the left-hand side of (i) tends to $\left(V-e^{i \lambda}\right)^{4}\left(V-e^{i \mu}\right)^{4} x$. Thus

$$
\left(V-e^{i \lambda}\right)^{4}\left(V-e^{i \mu}\right)^{4} x=\lim J\left(\lambda_{2}, \mu_{1}\right) x+J\left(\mu_{2}, \lambda_{1}\right) x .
$$

By Theorem 2.4,

$$
\sigma\left(J\left(\lambda_{2}, \mu_{1}\right) x\right) \subseteq\left[\lambda_{2}, \mu_{1}\right] \subseteq[\lambda, \mu]
$$

and

$$
\sigma\left(J\left(\mu_{2}, \lambda_{1}\right) x\right) \subseteq\left[\mu_{2}, \lambda_{1}\right] \subseteq[\lambda, \mu]^{\prime} .
$$

Hence any vector in the closure of the range of $\left(V-e^{i \lambda}\right)^{4}\left(V-e^{i \mu}\right)^{4}$ satisfies the conclusion of the lemma. But the range of the above operator is dense in the space; hence the conclusion holds for every vector in the space.

We are now in a position to define the system $\left\{G_{\lambda}, F_{\lambda}\right\}$ of resolving manifolds. For $0 \leqq \lambda \leqq 2 \pi$, set

$$
G_{\lambda}=\{x: \sigma(x) \subseteq[0, \lambda]\} \text {, and } F_{\lambda}=\{x: \sigma(x) \subseteq[\lambda, 2 \pi]\} \text {. }
$$

3.2 THEOREM. For $0 \leqq \lambda \leqq 2 \pi$, the resolving manifolds have the following properties:

(a) $G_{\lambda}$ and $F_{\lambda}$ are closed linear manifolds;

(b) $G_{\lambda}$ and $F_{\lambda}$ have only the zero element in common;

(c) for $0 \leqq \lambda \leqq \mu \leqq 2 \pi, G_{\lambda} \leqq G_{\mu}$ and $F_{\mu} \leqq F_{\lambda}$;

(d) the pair $\left\{G_{\lambda}, F_{\lambda}\right\}$ span the space $X$; and

(e) $G_{\lambda}$ and $F_{\lambda}$ reduces $V$.

Proof. Part (a) follows from Theorem 2.5. If $x$ is in the intersection of $G_{\lambda}$ and $F_{\lambda}$, then $\sigma(x) \subseteq\left\{e^{i \lambda}\right\} \cup\left\{e^{i 0}\right\}$. Thus $\sigma(x)$ consists of at most two points; hence, as was shown in the proof of Theorem 2.7, $x=0$. For part (c), suppose $\lambda$ is zero, then $G_{0}=\left\{x: \sigma(x) \cong\left\{e^{i 0}\right\}\right.$. But then $G_{0}=(0)$. The same argument shows that $F_{2 \pi}=(0)$. It is obvious that $G_{2 \pi}=X$ and $F_{0}=X$. If $0<\lambda<\mu<2 \pi$, then $[0, \lambda] \leqq$ $[0, \mu]$ and so $(0)=G_{0} \subseteq G_{\lambda} \subseteq G_{\mu} \subseteq G_{2 \pi}=X$. In the same way $X=$ $F_{0} \supseteqq F_{\lambda} \supseteqq F_{\mu} \supseteqq F_{2 \pi}=(0)$. Since $[0, \lambda]^{\prime} \cong[\lambda, 2 \pi]$, we have

$$
\begin{array}{cc}
\{x+y: \sigma(x) \cong[0, \lambda], & \left.\sigma(y) \cong[0, \lambda]^{\prime}\right\} \\
\subseteq\{x+y: \sigma(x) \subseteq[0, \lambda], & \sigma(y) \cong[\lambda, 2 u]\} .
\end{array}
$$


Taking the closure of both sides, and noting that, by Lemma 3.1, the closure of the left-hand side is $X$, proves part (d). Part (e) will be proved, once it is shown that $\sigma(V x) \subseteq \sigma(x)$; but this follows from the equation $R(z) V x=V R(z) x, z$ in $\rho(V)$. Since $V$ is a bounded operator, the right-hand side has an analytic extension to $\rho(x)$; hence $\rho(V x) \supseteqq$ $\rho(x)$ or $\sigma(V x) \subseteq \sigma(x)$.

The following theorem characterizes the spectrum of $V$ in terms of the behavior of $G_{\lambda}$ as a function of $\lambda$. Recall that we have, by hypotheses, ruled out the possibility of point or residual spectrum.

3.3 THEOREM. (a) The point $e^{i \lambda}$ is in $\rho(V)$ if and only if there exists an $\varepsilon>0$ such that $G_{\mu}=G_{\lambda}$ for all $\mu$ such that $\lambda-\varepsilon<\mu<$ $\lambda+\varepsilon$.

(b) The point $e^{i \lambda}$ is in $\sigma(V)=\sigma_{c}(V)$ if and only if $G_{\mu}$ is not constant in any neighborhood of $\lambda$.

Proof. If $\lambda<\mu$ then $(\lambda, \mu)$ will denote the open subarc of the circumference of the unit circle from $e^{i \lambda}$ to $e^{i \mu}$.

(a) Suppose $e^{i \lambda}$ is in $\rho(V)$, then there exists an $\varepsilon>0$ such that if $\lambda-\varepsilon<\mu<\lambda+\varepsilon$, then $e^{i \mu}$ is in $\rho(V)$. If $\mu$ is such that $\lambda-\varepsilon<$ $\mu<\lambda$, then $G_{\mu} \subseteq G_{\lambda}$; but if $x$ is in $G_{\lambda}$, then since $(\lambda-\varepsilon, \lambda) \subset \rho(V)$, we have $\sigma(x) \leqq[0, \lambda-\varepsilon] \subset[0, \mu]$. Hence, $x$ is in $G_{\mu}$ and $G_{\mu}=G_{\lambda}$ for $\lambda-\varepsilon<\mu<\lambda$. The same argument is used for $\lambda<\mu<\lambda+\varepsilon$. On the other hand, suppose that for some $\varepsilon>0, G_{\mu}=G_{\lambda}$ for $\lambda-\varepsilon<$ $\mu<\lambda+\varepsilon$. It must be shown that the open arc $(\lambda-\varepsilon, \lambda+\varepsilon)$ lies in $\rho(V)$. Let $\mu_{1}$ and $\mu_{2}$ be any two numbers such that $\lambda-\varepsilon<\mu_{1}<$ $\mu_{2}<\lambda+\varepsilon$. We shall first show that $J\left(\mu_{1}, \mu_{2}\right) x=0$ for each $x$ in $X$. By Theorem 2.4, we know that for any $x$ in $X$, we have $\sigma\left(J\left(\mu_{1}, \mu_{2}\right) x\right) \subseteq$ $\left[\mu_{1}, \mu_{2}\right]$; hence if $\mu_{2}<s<\lambda+\varepsilon$, then $J\left(\mu_{1}, \mu_{2}\right) x$ is in $G_{s}$ because $\left[\mu_{1}, \mu_{2}\right] \subset[0, s]$. Now if $\lambda-\varepsilon<\mu<\mu_{1}$, then by assumption $G_{\mu}=G_{s}$; hence $J\left(\mu_{1}, \mu_{2}\right) x$ belongs to $G_{\mu}$ which implies that $\sigma\left(J\left(\mu_{1}, \mu_{2}\right) x\right) \subseteq[0, \mu]$. Thus $\sigma\left(J\left(\mu_{1}, \mu_{2}\right) x\right) \subseteq\left[\mu_{1}, \mu_{2}\right] \cap[0, \mu]=\varnothing$. By part (i) of Lemma 2.3, we have $J\left(\mu_{1}, \mu_{2}\right) x=0$. Using the operational calculus and splitting the contour in the usual way, we have

$$
\left(V-e^{i \mu_{1}}\right)^{2}\left(V-e^{i \mu_{2}}\right)^{2} x=J\left(\mu_{1}, \mu_{2}\right) x+J\left(\mu_{2}, \mu_{1}\right) x=J\left(\mu_{2}, \mu_{1}\right) x .
$$

Thus

$$
\sigma\left(\left(V-e^{i \mu_{1}}\right)^{2}\left(V-e^{i \mu_{2}}\right)^{2} x\right)=\sigma\left(J\left(\mu_{2}, \mu_{1}\right) x\right) \leqq\left[\mu_{2}, \mu_{1}\right] .
$$

But since (cf. [2; p. 589])

$$
\sigma(x) \sqsubseteq \sigma\left(\left(V-e^{i \mu_{1}}\right)^{2}\left(V-e^{i \mu_{2}}\right)^{2} x\right) \cup\left\{e^{i \mu_{1}}\right\} \cup\left\{e^{i \mu_{2}}\right\},
$$

we have $\sigma(x) \subseteq\left[\mu_{2}, \mu_{1}\right]$. Thus for any $x$ in $X$, we have $\left(\mu_{1}, \mu_{2}\right) \subset \rho(x)$ 
where $\left(\mu_{1}, \mu_{2}\right)$ is any open subarc of $(\lambda-\varepsilon, \lambda+\varepsilon)$. Thus for any $x$ in $X$ and any point $e^{i \mu}$, with $\lambda-\varepsilon<\mu<\lambda+\varepsilon$, we have $e^{i \mu}$ in $\rho(x)$ and $\left(e^{i \mu}-V\right) x\left(e^{i \mu}\right)=x$; hence $\left(e^{i \mu}-V\right)$ maps $X$ onto $X$, and so $e^{i \mu}$ is in $\rho(V)$ for each $\mu$ in $(\lambda-\varepsilon, \lambda+\varepsilon)$.

(b) This is obvious since $\sigma(V)=\sigma_{c}(V)$.

4. Weakly almost periodic operators. In this section we shall show how the methods developed by Dunford can be applied to a class of operators studied by E. R. Lorch [6]. The main results presented here do not differ from those in the paper by Lorch, but the manifolds defined here are larger than those in [6].

Let $X$ be a reflexive Banach space and $V$ an invertible bounded operator such that

$$
\left\|V^{n}\right\| \leqq K \text { for some constant } K \text { and } n= \pm 1, \pm 2 \cdots .
$$

Lorch calls an operator $V$ in a reflexive Banach space which satisfies condition (a') a weakly almost periodic (w.a.p.) operator. Since condition $\left(a^{\prime}\right)$ is more stringent than condition (a), we see from Theorem 2.1 that the spectrum of a w.a.p. operator is contained in the circumference of the unit circle. The following lemma is basic to the discussion; its proof may be found in [5].

4.1 Lemma. For each $\xi$ on the circumference of the unit circle there exists a bounded projection $P_{\xi}$ with range $R\left(P_{\xi}\right)=\{x: V x=\xi x\}$ and null space $N\left(P_{\xi}\right)=\operatorname{cl}(V-\xi)(X)$. Moreover, the space $X$ is the direct sum of the subspaces $R\left(P_{\xi}\right)$ and $N\left(P_{\xi}\right)$.

If $\xi-V$ is one-to-one for some $\xi$ on the circumference of the unit circle, then from the above lemma, $R\left(P_{\xi}\right)=(0)$; hence the range of $\xi-V$ is dense in $X$. Thus $\xi$ is in the continuous spectrum; hence the residual spectrum of a w.a.p. operator is void. Furthermore, condition $\left(\mathrm{a}^{\prime}\right)$ implies that for $|z|>1$,

$$
\|R(z ; V)\|=\left\|\sum_{k=0}^{\infty} z^{-k-1} V^{k}\right\| \leqq K(|z|-1)^{-1}
$$

and for $|z|<1$,

$$
\|R(z ; V)\|=\left\|-\sum_{k=0}^{\infty} z^{k} V^{-k-1}\right\| \leqq K(1-|z|)^{-1} ;
$$

hence a w.a.p. operator satisfies the stronger condition of having a resolvent with a first order rate of growth. Thus Theorem 2.4 applies to a w.a.p. operator with $J\left(\lambda_{1}, \lambda_{2}\right)$ now defined by:

$$
J\left(\lambda_{1}, \lambda_{2}\right)=\frac{1}{2 \pi i} \int_{o\left(\lambda_{1}, \lambda_{2}\right)} F(z)\left(z-e^{i \lambda_{1}}\right)\left(z-e^{i \lambda_{2}}\right) R(z) d z .
$$


The set of all $x$ whose spectrum lies in a closed subarc of the circumference of the unit circle is a closed linear manifold since this is a consequence of the finite order of the rate of growth of the resolvent. In the case of a w.a.p. operator, Theorem 2.7 is replaced by the following theorem.

4.2 THEOREM. Let $\xi_{1}, \xi_{2}, \cdots, \xi_{n}$ be points on the circumference of the unit circle and let $W$ denote the range of the operator $\left(V-\xi_{1}\right)\left(V-\xi_{2}\right) \cdots\left(V-\xi_{n}\right)$; then

(i) $X=R\left(P_{\xi_{1}}\right) \oplus \cdots \oplus R\left(P_{\xi_{n}}\right) \oplus \mathbf{c l}(W)$, and

(ii) for any vector $y$ in $W$ there is a unique decomposition $y=$ $y_{1}+y_{2}+\cdots+y_{n}$ where $\sigma\left(y_{k}\right) \leqq\left[\xi_{k}, \xi_{k+1}\right], k=1,2, \cdots, n$ with $\xi_{n+1}=\xi_{1}$.

Proof. In part (i), the general case is handled by induction. The case $n=2$ follows from the relation

$$
I=P_{\xi_{1}}+P_{\xi_{2}}+\left(I-P_{\xi_{1}}\right)\left(I-P_{\xi_{2}}\right)
$$

together with the result (cf. [2; p. 592]) that the range of the projection $\left(I-P_{\xi_{1}}\right)\left(I-P_{\xi_{2}}\right)$ is equal to the closure of the range of the operator $\left(V-\xi_{1}\right)\left(V-\xi_{2}\right)$.

The proof of part (ii) is identical to the proof of Theorem 2.7 (ii).

If $\xi_{1}, \xi_{2}, \cdots, \xi_{n+1}=\xi_{1}$ is any finite collection of points on the circumference of the unit circle, then this collection forms a partition of the circumference into a finite number of nonoverlapping closed intervals $\left[\xi_{k}, \xi_{k+1}\right]$. By the preceding theorem, any vector $x$ in the space may be approximated by a vector of the form $x_{1}+\cdots+x_{n}+$ $y_{1}+\cdots+y_{n}$ where $V x_{k}=\xi_{k} x_{k}$ and $\sigma\left(y_{k}\right) \subseteq\left[\xi_{k}, \xi_{k+1}\right], k=1,2, \cdots, n$. An obvious question as to the character of $\sigma\left(x_{k}\right)$ is answered by the following lemma.

4.3 Lemma. For any $\xi=e^{i \lambda}$, then $V x=\xi x, x \neq 0$, if and only if $\sigma(x)=\{\xi\}$.

Proof. If $x \neq 0$ and $V x=\xi x$, then

$$
R(z) x=\sum_{k=0}^{\infty}(z-\xi)^{-k-1}(V-\xi)^{k} x=(z-\xi)^{-1} x ;
$$

thus $R(z) x$ is analytic for $z \neq \xi$, and so $\sigma(x)=\{\xi\}$.

On the other hand, if $\sigma(x)=\{\xi\}$, then $\sigma(x) \neq \varnothing$ and so $x \neq 0$. If $\xi=e^{i \lambda}$, choose two sequences $\left\{\lambda_{n}\right\}$ and $\left\{\mu_{n}\right\}$ such that $\lambda_{n}<\lambda<\mu_{n}$ with $\lambda_{n}$ and $\mu_{n}$ both tending to $\lambda$. Using the operational calculus and splitting the contour in the usual way, we have

$$
\left(V-e^{i \lambda_{n}}\right)\left(V-e^{i \mu_{n}}\right) x=J\left(\lambda_{n}, \mu_{n}\right) x+J\left(\mu_{n}, \lambda_{n}\right) x .
$$


Since $\sigma(x)=\{\xi\}, J\left(\mu_{n}, \lambda_{n}\right) x=0$ for every $n$. By Theorem 2.4, $J\left(\lambda_{n}, \mu_{n}\right) x$ tends to zero as $n$ tends to infinity. Thus

$$
(V-\xi)^{2} x=\lim _{n}\left(V-e^{i \lambda_{n}}\right)\left(V=e^{i \mu_{n}}\right) x=\lim _{n} J\left(\lambda_{n}, \mu_{n}\right) x=0 .
$$

Thus $(z-\xi) R(z) x=x+(z-\xi)^{-1}(V-\xi) x$. Since $(z-\xi) R(z) x$ is bounded as $z$ tends to $\xi$ along the transversal through $\xi$, we have $(V-\xi) x=0$.

Theorem 2.9 relied mainly on two hypotheses. The first was that for any finite set of points $\xi_{1}, \xi_{2}, \cdots, \xi_{n}$ each vector $x$ could be approximated by $n$ vectors $y_{1}, y_{2}, \cdots, y_{n}$ where $\sigma\left(y_{k}\right) \subseteq\left[\xi_{k}, \xi_{k+1}\right]$. In the case of a w.a.p. operator, this hypothesis was shown to be valid in the discussion preceding Lemma 4.3 together with the lemma itself. The second hypothesis was provided by Lemma 2.8 which said that

for any $\varepsilon>0$ there exists a number $A_{\varepsilon}$ such that for any $x$ in $X$, we have $\|(V-I) x\| \leqq(3 / 4) \varepsilon\|x\|+A_{\varepsilon}\left\|(V-I)^{4} x\right\|$.

The validity of $(\beta)$ depended on the condition $\left\|V^{n}\right\|=o(n)$, and it is easily seen from the proof of Lemma 2.8 that $(\beta)$ remains true if the fourth power is replaced by any lower power. Since a w.a.p. operator satisfies the condition $\left\|V^{n}\right\|=o(n),(\beta)$ holds for a w.a.p. operator with the fourth power replaced by the second power. With the above discussion in mind, Theorem 2.9 holds for a w.a.p. operator, and can be proved in exactly the same way that Theorem 2.9 was proved.

In order to obtain a system of resolving manifolds for a w.a.p. operator, the following two lemmas are needed. The first lemma is a special case of a more general result due to Dunford (cf. [2: p. 593]). The second lemma will be proved by applying techniques developed by Dunford.

4.4 Lemma. Let $\xi_{1}=e^{i \lambda_{1}}$ and $\xi_{2}=e^{i \lambda_{2}}$ be any two points with $\lambda_{1}<\lambda_{2}$, and denote the open subarc $\left\{e^{i \lambda}: \lambda \notin\left[\lambda_{1}, \lambda_{2}\right]\right\}$ by $\left[\lambda_{1}, \lambda_{2}\right]^{\prime}$. Then the set of all vectors of the form $x+y$ with $\sigma(x) \subseteq\left[\lambda_{1}, \lambda_{2}\right]$ and $\sigma(y) \subseteq$ $\left[\lambda_{1}, \lambda_{2}\right]^{\prime}$ is dense in $X$.

4.5. Lemma. If $\sigma(x)=\left\{\xi_{1}\right\} \cup\left\{\xi_{2}\right\}$, then $x=x_{1}+x_{2}$ where $V x_{1}=$ $\xi_{1} x_{1}$ and $V x_{2}=\xi_{2} x_{2}$.

Proof. Suppose $\sigma(x)=\left\{\xi_{1}\right\} \cup\left\{\xi_{2}\right\}$, let $[x]$ denote the closed linear manifold generated by $R(z) x$ as $z$ ranges over $\rho(V)$. This subspace is defined in [2; p. 564] and the following properties are given there.

(a) $V([x]) \subseteq[x]$;

(b) $x$ is in $[x]$;

(c) if $y$ is in $[x]$ then $[y] \subseteq[x]$; 
(d) if $V_{x}$ denotes the restriction of $V$ to the subspace $[x]$, then $\sigma\left(V_{x}\right)=\sigma(x)$.

From (d), $\sigma\left(V_{x}\right)=\left\{\xi_{1}\right\} \cup\left\{\xi_{2}\right\}$; hence $\left\{\xi_{1}\right\}$ and $\left\{\xi_{2}\right\}$ are spectral sets for $V_{x}$. Let $X_{i}$ be the range of the projection $E_{i}$ associated with the spectral set $\left\{\xi_{i}\right\}$ and $V_{i}$ the restriction of $V_{x}$ to the subspace $X_{i}$ for $i=1$, 2. Then $\sigma\left(V_{i}\right)=\left\{\xi_{i}\right\}$ and $[x]=X_{1} \oplus X_{2}$. Since $x$ is in $[x], x$ is of the form $x_{1}+x_{2}$ where $x_{1}$ is in $X_{1}$ and $x_{2}$ is in $X_{2}$. On applying Lemma 4.3 to these subspaces, we obtain the assertion of the lemma.

Using the preceding lemmas, we can define a system to resolving manifolds for a w.a.p. operator. Let $G_{2 \pi}=X$ and $F_{2 \pi}=(0)$; for $0 \leqq$ $\lambda<2 \pi$, let $G_{\lambda}$ be the set of all $x$ such that $\sigma(x) \cong[0, \lambda]$ together with $P x=0$ and $P_{\lambda} x=0$. Let $F_{\lambda}$ be the set of all $x$ for which $\sigma(x) \subseteq[\lambda, 2 \pi]$.

4.6 THEOREM. For $0 \leqq \lambda \leqq 2 \pi$, the resolving manifolds satisfy

(a) $G_{\lambda}$ and $F_{\lambda}$ are closed linear manifolds,

(b) $G_{\lambda}$ and $F_{\lambda}$ have only the zero element in common,

(c) for $0 \leqq \lambda<\mu \leqq 2 \pi, G_{\lambda} \leqq G_{\mu}$ and $F_{\mu} \leqq F_{\lambda}$;

(d) the pair $\left\{G_{\lambda}, F_{\lambda}\right\}$ spans $X$; and

(e) $G_{\lambda}$ and $F_{\lambda}$ reduce $V$.

Proof. If $\xi=e^{i \lambda}$, then $G_{\lambda}=\{x: \sigma(x) \subseteq[0, \lambda]\} \cap N\left(P_{\lambda}\right) \cap N\left(P_{1}\right)$. The fact that $\{x: \sigma(x) \subseteq[0, \lambda]\}$ is a closed linear manifold follows directly from Theorem 2.5 since the validity of that theorem depended only on the finite rate of growth of the resolvent. This last statement also applies to $F_{\lambda}$. If $x$ is in both $G_{\lambda}$ and $F_{\lambda}$, then $\sigma(x) \cong$ $\{1\} \cup\{\xi\}$, by Lemma $4.5, x$ is of the form $x_{1}+x_{2}$ where $P x_{1}=x_{1}$ and $P_{\lambda} x_{2}=x_{2}$. Since $x$ is in $G_{\lambda}, P x$ and $P_{\lambda} x$ are both 0 ; hence $x_{1}=P x_{1}=$ $P x=0$, and $x_{2}=P_{\lambda} x_{2}=P_{\lambda} x=0$. Thus $x=0$. For part (c), suppose $\lambda=0$, then $G_{0}=\left\{x: \sigma(x) \subseteq\left\{e^{i 0}\right\}, P x=0\right\}$. If $x$ is in $G_{0}$ with $x \neq 0$, then by Lemma 4.3, we have $P x=x$. Since $x$ is in $G_{0}$, it follows that $P x=0$. Hence, contrary to the hypothesis, $x$ is zero. If $\lambda$ is not zero, and $\lambda$ is less that $\mu$, then if $x$ is in $G_{\lambda}$, we have $\sigma(x) \subseteq$ $[0, \lambda] \subset[0, \mu], P x=0$, and $P_{\lambda} x=0$. To show that $x$ is in $G_{\mu}$, we need only show that $P_{\mu} x=0$. Let $x_{1}=P_{\mu} x$ and suppose $x_{1}$ is not zero. By Lemma 4.1, $x=x_{1}+h$ where $h$ is in the closure of the range of $\left(V-e^{i \mu}\right)$. Since $x_{1}$ is an eigenvector, $x_{1}(z)=\left(z-e^{i \mu}\right)^{-1} x_{1}$, and by Lemma 2.3, $x(z)=x_{1}(z)+h(z)$ for $z$ in $\rho(h), z \neq e^{i \mu}$. Since $x_{1}(z)$ is in the range of $P_{\mu}, h(z)$ is in the null space of $P_{\mu}$; hence the singularity of $x_{1}(z)$ at $z=e^{i \mu}$ cannot be cancelled out by $h(z)$. Thus $e^{i \mu}$ is in $\sigma(x)$. But $\sigma(x) \subseteq[0, \lambda]$ and $e^{i \mu}$ is not in $[0, \lambda]$; thus $P_{\mu} x$ must be zero, and $G_{\lambda} \subseteq G_{\mu}$ for $\lambda$ less than $\mu$. The monotonicity of $F_{\lambda}$ is obvious. Part (d) is a direct consequence of Lemma 4.4. Since $V P_{\lambda}=P_{\lambda} V$, part (e) 
is proved in exactly the same manner as part (e) of Theorem 3.2.

4.7 TheOREM. (a) The point $e^{i \lambda}$ is in the point spectrum of $V$ if and only if $G_{\lambda}$ is properly contained in the intersection of $G_{\mu}$ for $\mu>\lambda$.

(b) The point $e^{i \lambda}$ is in the resolvent set if and only if there exists an $\varepsilon>0$ such that $G_{\mu}=G_{\lambda}$ for $\lambda-\varepsilon<\mu<\lambda+\varepsilon$.

(c) The point $e^{i \lambda}$ is in the continuous spectrum if and only if $G_{\lambda}$ is the intersection of $G_{\mu}$ for $\mu>\lambda$ and $G_{\mu}$ is not constant in any neighborhood of $\lambda$.

Proof. If $e^{i \lambda}$ is in the point spectrum, then there exists a vector $x_{0} \neq 0$ such that $V x_{0}=e^{i \lambda} x_{0}$; hence $P_{\lambda} x_{0}=x_{0}$. By Lemma 4.3, $\sigma\left(x_{0}\right)=$ $\left\{e^{i \lambda}\right\}$, and since $P_{\mu} P_{\lambda}=0$ for $\mu \neq \lambda$, we have $P_{\mu} x_{0}=0$ for $\mu \neq \lambda$. Hence, if $\mu$ is greater than $\lambda$, we have $\sigma\left(x_{0}\right)=\left\{e^{i \lambda}\right\} \subset[0, \mu], P_{\mu} x_{0}=0$, and $P x_{0}=0$, i.e., $x_{0}$ is in $G_{\mu}$ for $\mu$ greater than $\lambda$. Since $P_{\lambda} x_{0}=$ $x_{0} \neq 0$, we see that $x_{0}$ is not in $G_{\lambda}$; thus $G_{\lambda}$ is properly contained in the intersection of $G_{\mu}$ for $\mu>\lambda$.

On the other hand, suppose $G_{\lambda}$ is properly contained in the intersection of $G_{\mu}$ for $\mu>\lambda$, then let $x$ be in this intersection, but not in $G_{\lambda}$. We then have $P x=0$ and $\sigma(x) \cong[0, \mu]$ for all $\mu$ greater than $\lambda$; hence, $\sigma(x) \leqq[0, \lambda]$. If $P_{\lambda} x$ were zero, then $x$ would be in $G_{\lambda}$; thus $P_{\lambda} x \neq 0$ and $V\left(P_{\lambda} x\right)=e^{i \lambda} P_{\lambda} x$ showing that $e^{i \lambda}$ is in the point spectrum.

Parts (b) and (c) are proved in almost exactly the same way that Theorem 3.2 was proved.

\section{BIBLIOGRAPHY}

1. N. Dunford, Spectral Theory, I. Convergence to projections, Trans. Amer. Math. Soc., 54 (1943), 185-217.

2. - Spectral Theory, II. Resolutions of the identity, Pacific J. Math., 2 (1952), 559-614.

3. - Spectral operators, Pacific J. Math., 4 (1954), 321-354.

4. N. Dunford and J. T. Schwartz, Linear operators, vol. II, Interscience Pub. Co., New York, to appear.

5. E. R. Lorch, Means of iterated transformations in reflexive vector spaces, Bull. Amer. Math. Soc., 45 (1939), 945-947.

6. - The integral representation of weakly almost periodic transformations in reflexive vector spaces, Trans. Amer. Math. Soc., 49 (1941), 18-40.

7. A. E. Taylor, Introduction to functional analysis, John Wiley and Sons, New York, 1958.

8. J. Wermer, The existence of invariant subspaces, Duke Math. J., 19 (1952), 615-622.

9. F. Wolf, Operators in Banach space which admit a generalized spectral decomposition, Proc. Kon. Ned. Akad. v. Wet. 60 (=Ind. Math. 19) (1957), 302-311. 



\section{PACIFIC JOURNAL OF MATHEMATICS}

\section{EDITORS}

\section{RalPh S. Phillips}

Stanford University

Stanford, California

M. G. Arsove

University of Washington

Seattle 5, Washington
J. Dugundji

University of Southern California Los Angeles 7, California

Lowell J. Paige

University of California

Los Angeles 24, California

\section{ASSOCIATE EDITORS}
E. F. BECKENBACH
D. DERRY
H. L. ROYDEN
E. G. STRAUS
T. M. CHERRY
M. OHTSUKA
E. SPANIER
F. WOLF

\section{SUPPORTING INSTITUTIONS}

\author{
UNIVERSITY OF BRITISH COLUMBIA \\ CALIFORNIA INSTITUTE OF TECHNOLOGY \\ UNIVERSITY OF CALIFORNIA \\ MONTANA STATE UNIVERSITY \\ UNIVERSITY OF NEVADA \\ NEW MEXICO STATE UNIVERSITY \\ OREGON STATE UNIVERSITY \\ UNIVERSITY OF OREGON \\ OSAKA UNIVERSITY \\ UNIVERSITY OF SOUTHERN CALIFORNIA
}

STANFORD UNIVERSITY

UNIVERSITY OF TOKYO

UNIVERSITY OF UTAH

WASHINGTON STATE UNIVERSITY

UNIVERSITY OF WASHINGTON

AMERICAN MATHEMATICAL SOCIETY

CALIFORNIA RESEARCH CORPORATION SPACE TECHNOLOGY LABORATORIES

NAVAL ORDNANCE TEST STATION

Printed in Japan by International Academic Printing Co., Ltd., Tokyo Japan 


\section{Pacific Journal of Mathematics}

\section{Vol. 13, No. 1 \\ March, 1963}

Frantz Woodrow Ashley, Jr., A cone of super-(L) functions............. 1

Earl Robert Berkson, Some metrics on the subspaces of a Banach space....

Felix Earl Browder and Walter Strauss, Scattering for non-linear wave

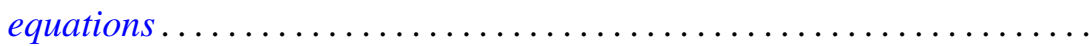

Edmond Darrell Cashwell and C. J. Everett, Formal power series ..........

Frank Sydney Cater, Continuous linear functionals on certain topological

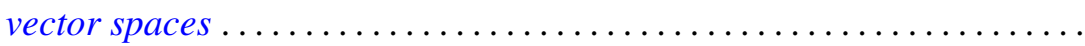

John Douglas Dixon, General group extensions ....................

Robert Pertsch Gilbert, On harmonic functions of four variables with

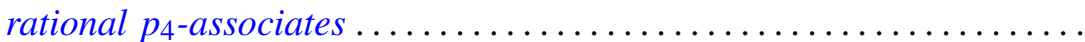

Irving Leonard Glicksberg, On convex hulls of translates ..............

Simon Hellerstein, On a class of meromorphic functions with deficient zeros

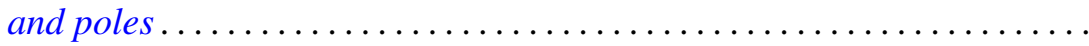

Donald William Kahn, Secondary cohomology operations which extend the

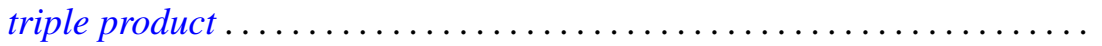

G. K. Leaf, A spectral theory for a class of linear operators .............

R. Sherman Lehman, Algebraic properties of the composition of solutions of partial differential equations ........................... 157

Joseph Lehner, On the generation of discontinuous groups ............. 169

S. P. Lloyd, On certain projections in spaces of continuous functions ...... 171 Fumi-Yuki Maeda, Generalized spectral operators on locally convex spaces ..................................

Donald Vern Meyer, $E^{3}$ modulo a 3-cell

William H. Mills, An application of linear programming to permutation groups.

Richard Scott Pierce, Centers of purity in abelian groups

Christian Pommerenke, On meromorphic starlike functions ...

Zalman Rubinstein, Analytic methods in the study of zeros of

polynomials...

B. N. Sahney, On the Nörlund summability of Fourier series

Tôru Saitô, Regular elements in an ordered semigroup . .

Lee Meyers Sonneborn, Level sets on spheres...........

Charles Andrew Swanson, Asymptotic estimates for limit point

problems .

Lucien Waelbroeck, On the analytic spectrum of Arens . .

Alvin (Murray) White, Singularities of a harmonic function of three

variables given by its series development .............

Kōichi Yamamoto, Decomposition fields of difference sets ...

Chung-Tao Yang, On the action of $\mathrm{SO}(3)$ on a cohomology manifold... 